Review began 10/25/2021 Review ended 10/25/2021 Published 10/26/202 Exp. concern 04/07/2022

\section{Copyright 2021}

Sultan et al. This is an open access article distributed under the terms of the Creative Commons Attribution License CC-BY 4.0. which permits unrestricted use, distribution, and reproduction in any medium, provided the original author and source are credited.

\title{
Gastric Volvulus: A Rare Etiology of Acute Chest Pain
}

Maryam A. Sultan ${ }^{1}$, Alhanouf A. Hakami ${ }^{1}$, Meshari I. Alshabri ${ }^{2}$, Shahad A. Alsuwailem ${ }^{3}$, Nuwayyir E. Aqeel ${ }^{1}$, Rajis N. Aldosari ${ }^{4}$, Tariq H. Alrazhi ${ }^{5}$, Khaled A. Al-Wesabi ${ }^{6}$, Abdullah A. Alzayed ${ }^{7}$, Majed A. Alanazi ${ }^{8}$, Aioub S. Al Yousef ${ }^{9}$, Ahmed S. Alromaihi ${ }^{9}$, Roaa M. Aljadeed ${ }^{7}$, Sahar H. Alomar ${ }^{7}$, Malak Alshammari ${ }^{10}$

1. College of Medicine, Jazan University, Jizan, SAU 2. College of Medicine, King Saud University, Riyadh, SAU 3. College of Medicine, Alfaisal University, Riyadh, SAU 4. College of Medicine, Imam Mohammad Ibn Saud Islamic University, Riyadh, SAU 5. General Practice, Dhahran General Hospital, Dhahran, SAU 6. College of Medicine, Dar Al Uloom University, Riyadh, SAU 7. College of Medicine, King Saud Bin Abdulaziz University for Health Sciences, Riyadh, SAU 8. College of Medicine, Jordan University of Science and Technology, Irbid, JOR 9. College of Medicine, Arabian Gulf University, Manama, BHR 10. College of Medicine, Imam Abdulrahman Bin Faisal University, Dammam, SAU

Corresponding author: Malak Alshammari, saudidoctor2020@gmail.com

\section{Expression of Concern}

Expression of Concern date: April 07, 2022. Cite this expression of concern as Sultan M A, Hakami A A, Alshabri M I, et al. (April 07, 2022) Expression of Concern: Gastric Volvulus: A Rare Etiology of Acute Chest Pain. Cureus 14(4): x12. doi:10.7759/cureus.x12.

The concern relates to the provenance of this article as brought to our attention by Faisal Alhawaj, who denies authorship of this article and others published in Cureus. These articles were submitted and subsequently published purportedly as an effort coordinated by Imam Abdulrahman Bin Faisal University to ensure all medical interns publish at least one peer-reviewed article in order to qualify for enrollment in a postgraduate residency program as stipulated by The Saudi Commission for Health Specialties (SCFHS).

The journal has not been presented with enough evidence to warrant the formal retraction of these articles as both Imam Abdulrahman Bin Faisal University and The Saudi Commission for Health Specialties have failed to respond to numerous communications requesting additional information regarding these allegations. While we acknowledge that the provenance of these articles is very much in question, we cannot act until these claims have been investigated by the appropriate institutions with the results of said investigation communicated to Cureus.

The concern and this note will remain appended to the above-mentioned article until Cureus is provided with official confirmation from Imam Abdulrahman Bin Faisal University or The Saudi Commission for Health Specialties.

\begin{abstract}
Chest pain is one of the most common complaints in the emergency department. The etiologies of chest pain include a wide spectrum of conditions, ranging from mild and self-limited to life-threatening conditions. Gastrointestinal origins of acute chest pain, including esophageal, gastric, and biliary conditions, are not uncommon. We present the case of a 67-year-old man who presented to the emergency department with chest pain of three hours duration with pressure-like quality. The pain was associated with sweating, palpitation, nausea, and vomiting. The past medical history of the patient was remarkable for ischemic heart disease and percutaneous cardiac intervention. Both the electrocardiograph and cardiac biomarkers were normal. The patient experienced severe episodes of vomiting with one episode containing a small amount of blood. Upper gastrointestinal endoscopy revealed distorted anatomy of the stomach. Subsequently, the patient underwent a computed tomography scan which demonstrated an organoaxial gastric volvulus. The patient was resuscitated and underwent laparoscopic repair of the volvulus. Acute gastric volvulus is a very rare etiology of chest pain. Despite its rarity, physicians should keep a high index of suspicion of this condition after excluding the possible cardiac causes of chest pain.
\end{abstract}

Categories: Emergency Medicine, General Surgery

Keywords: operative laparoscopy, computed tomography (ct ), hematemesis, gastric volvulus, chest pain, case report

\section{Introduction}

Chest pain is one of the most common complaints in the emergency department. It is estimated that chest pain accounts for over seven million visits to the emergency department annually in the United States [1]. The etiologies of chest pain include a wide spectrum of conditions, ranging from mild and self-limited to life-threatening conditions. The initial evaluation of acute chest pain should include appropriate history, 
physical examination, electrocardiograph, and cardiac biomarkers in order to exclude the possibility of acute myocardial infarction. However, it should be remembered that less than $15 \%$ of patients with acute nontraumatic chest pain have acute coronary syndrome as the cause of their pain [2]. Gastrointestinal origins of acute chest pain, including esophageal, gastric, and biliary conditions, are not uncommon [3]. For instance, gastroesophageal reflux disease is the most common etiology of non-cardiac chest pain [4]. Here, we present the case of an elderly man with a history of ischemic heart disease who presented with sudden central chest pain with similar characteristics of cardiac chest pain. After thorough investigation, the patient was found as having an organoaxial gastric volvulus, which is a very rare clinical entity.

\section{Case Presentation}

We present the case of a 67-year-old man who presented to the emergency department with chest pain of three hours duration. He described the pain as pressure-like and was central in a location with no radiation. The pain started suddenly and remained constant. It was associated with sweating, palpitation, nausea, and vomiting. The pain was not relieved by simple analgesia medications. Further, it was exacerbated by exertion. The pain was not related to posture or respiration. He rated the pain as 8 out of 10 in severity. There was no change in urinary or bowel habits. The patient reported no history of anorexia, weight change, or fever.

The past medical history was remarkable for hypertension, diabetes mellitus, dyslipidemia, and stable angina. The patient underwent percutaneous coronary intervention five years ago. He remained symptomfree following the procedure. His medications included captopril $25 \mathrm{mg}$, metformin $1000 \mathrm{mg}$, aspirin $75 \mathrm{mg}$, clopidogrel $75 \mathrm{mg}$, and atorvastatin $20 \mathrm{mg}$. He was on regular follow-up visits with the cardiology team with no recent changes in his medications. The past surgical history was remarkable for open appendectomy. He was a retired school teacher. He never consumed alcohol and had a smoking history of 25 pack-years.

Upon examination, the patient appeared sick. He was not in respiratory distress. He was not pale, jaundiced, or cyanosed. His vital signs were a temperature of $36.8^{\circ} \mathrm{C}$, pulse rate of $110 \mathrm{bpm}$, blood pressure of $128 / 82$ $\mathrm{mmHg}$, and respiratory rate of $14 \mathrm{bpm}$. Cardiac examination revealed a normal S1 and S2 with no added sounds or murmurs. Respiratory examination revealed normal vesicular breathing throughout both lung fields. Abdominal examination revealed a soft and lax abdomen with epigastric tenderness. The electrocardiograph revealed a normal sinus rhythm with normal ST segments. The patient received soluble aspirin, clopidogrel, and sublingual nitroglycerin without clinical improvement. Both troponin-I and creatinine kinase enzymes were not elevated. The other basic laboratory investigation was within the normal limits (Table 1). 


\section{Cureus}

\begin{tabular}{|l|lll|}
\hline Laboratory investigation & Unit & Result & Reference range \\
\hline Hemoglobin & $\mathrm{g} / \mathrm{dL}$ & 13.1 & $13.0-18.0$ \\
\hline White blood cell & $1000 / \mathrm{mL}$ & 4.8 & $4.0-11.0$ \\
\hline Platelet & $1000 / \mathrm{mL}$ & 375 & $140-450$ \\
Erythrocyte sedimentation rate & $\mathrm{mm} / \mathrm{hr}$ & 13 & $0-20$ \\
\hline C-reactive protein & $\mathrm{mg} / \mathrm{dL}$ & 5.2 & $0.3-10.0$ \\
\hline Total bilirubin & $\mathrm{mg} / \mathrm{dL}$ & 1.1 & $0.2-1.2$ \\
\hline Albumin & $\mathrm{g} / \mathrm{dL}$ & 3.4 & $3.4-5.0$ \\
\hline Alkaline phosphatase & $\mathrm{U} / \mathrm{L}$ & 50 & $46-116$ \\
\hline Gamma-glutamyltransferase & $\mathrm{U} / \mathrm{L}$ & 48 & $15-85$ \\
\hline Alanine transferase & $\mathrm{U} / \mathrm{L}$ & 60 & $14-63$ \\
\hline Aspartate transferase & $\mathrm{U} / \mathrm{L}$ & 27 & $15-37$ \\
\hline Blood urea nitrogen & $\mathrm{mg} / \mathrm{dL}$ & 17 & $7-18$ \\
\hline Creatinine & $\mathrm{mg} / \mathrm{dL}$ & 1.2 & $0.7-1.3$ \\
\hline Sodium & $\mathrm{mEq} / \mathrm{L}$ & 138 & $136-145$ \\
\hline Potassium & $\mathrm{mEq} / \mathrm{L}$ & 4.5 & $3.5-5.1$ \\
Chloride & $\mathrm{mEg} / \mathrm{L}$ & 106 & $98-107$ \\
\hline
\end{tabular}

TABLE 1: Summary of the results of laboratory findings.

The patient experienced severe nausea with an episode of vomiting that contained a small amount of blood. Intravenous omeprazole was administered and resulted in improvement of his symptoms. Then, the patient was prepared for emergency upper gastrointestinal endoscopy which revealed distorted anatomy of the stomach with no gross lesions. Further, the endoscopist could not pass through the pyloric canal.

Subsequently, an abdominal computed tomography scan with oral contrast. The scan demonstrated thoracic herniation of the stomach with rotation about its long axis. The lesser curvature was inferior to the greater curvature. Such findings conferred the diagnosis of organoaxial gastric volvulus (Figures 1,2). 


\section{Cureus}

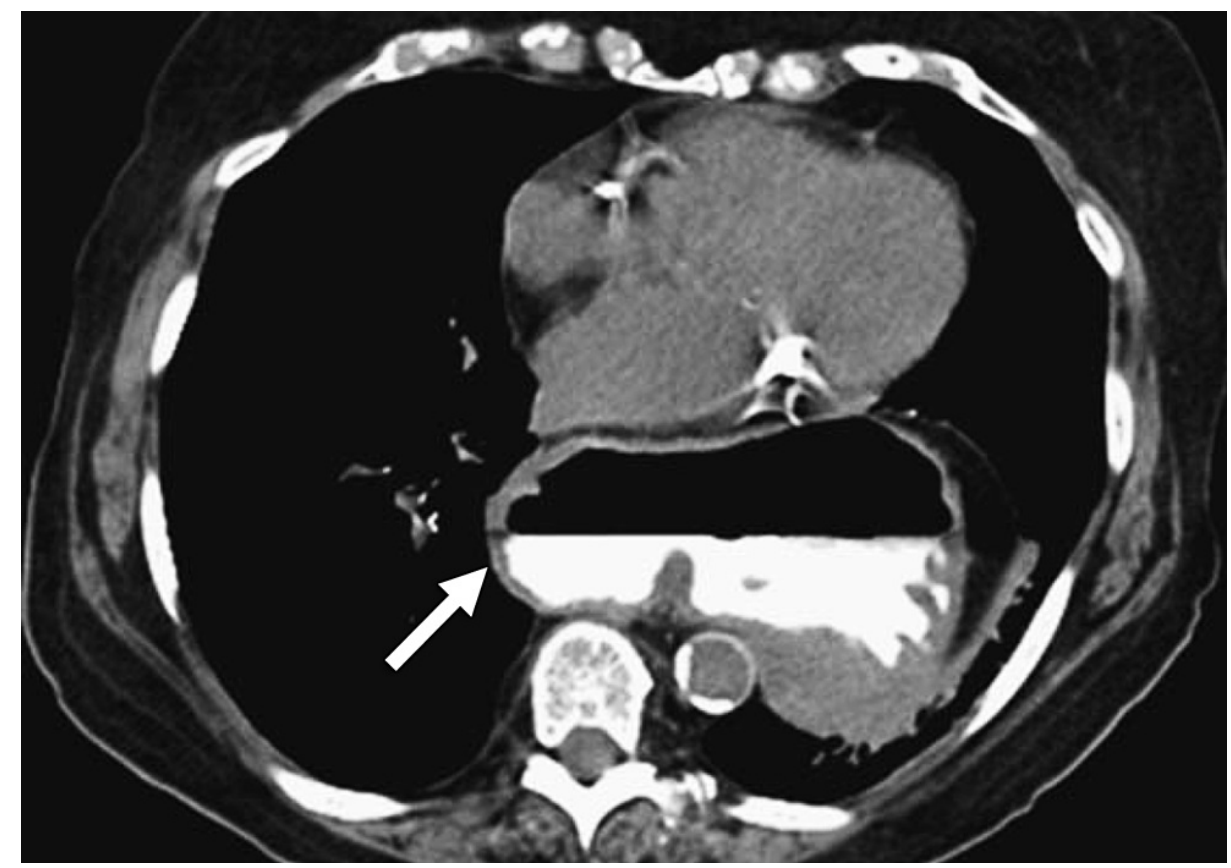

\section{FIGURE 1: Axial CT image.}

Selected computed tomography image of the chest in the axial plane demonstrating the contrast-filled stomach (arrow) herniated in the thoracic cavity.

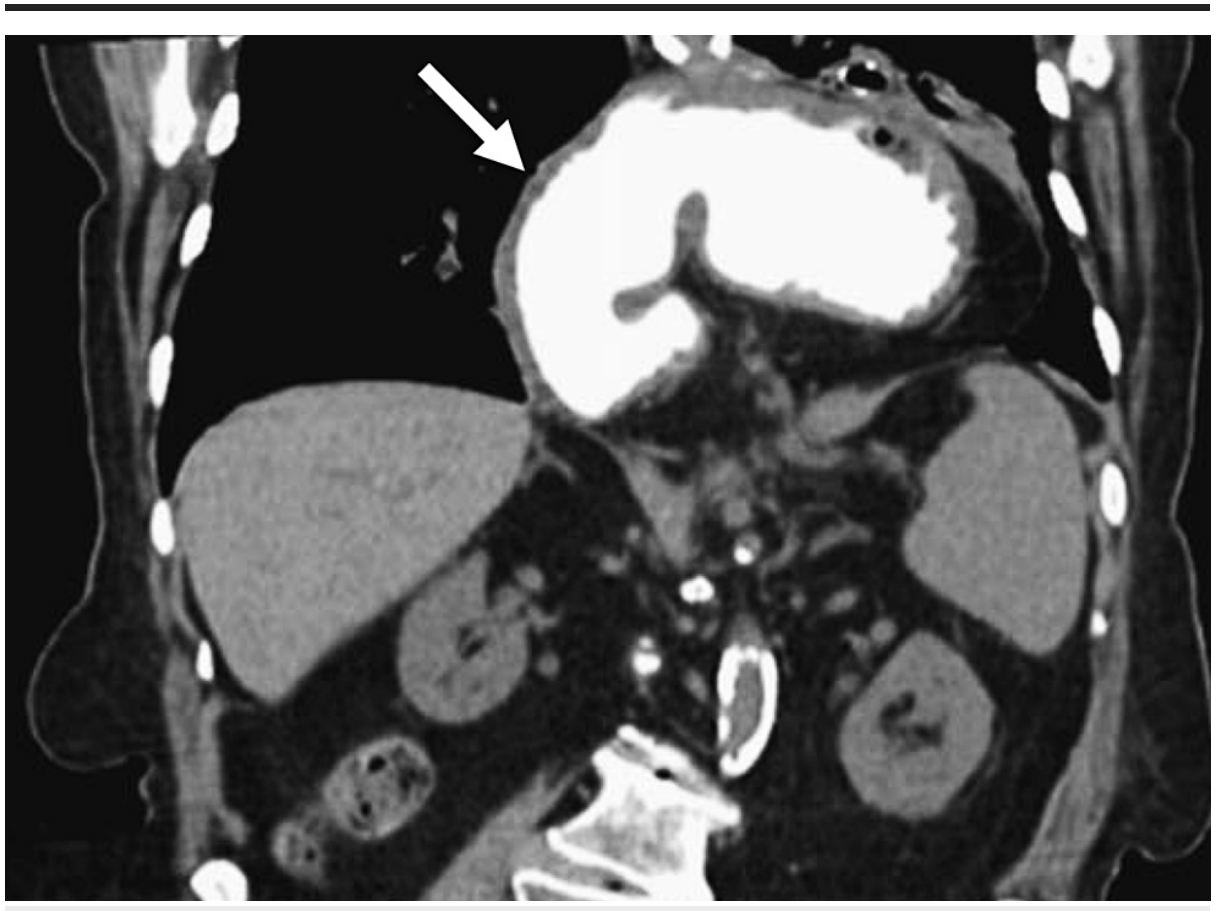

FIGURE 2: Coronal CT image.

Selected computed tomography image of the upper abdomen in the coronal plane demonstrating the contrastfilled stomach (arrow) rotated along its vertical axis, conferring the diagnosis of organoaxial gastric volvulus.

The patient was resuscitated with intravenous fluid and electrolyte therapy. Nasogastric tube decompression was performed. The decompression of the stomach resulted in reliving the patient's symptoms. The patient remained symptom-free and was hemodynamically stable. Two days later, the patient underwent laparoscopic surgery for the definitive treatment of the volvulus. The herniated stomach was reduced. Careful examination of the stomach showed no areas of ischemia. The stomach was detorted and gastropexy was performed. The patient tolerated the procedure with no complications. The total operative time was 150 
minutes and the estimated blood loss was $50 \mathrm{~mL}$. The patient had an uneventful recovery. He was discharged on the sixth postoperative day. He was followed up for one year and had no active issues.

\section{Discussion}

We presented the case of a 67-year-old man with gastric volvulus who presented with central crushing chest pain with normal electrocardiograph and cardiac biomarkers findings. Gastric volvulus is defined as the rotation of the stomach along its vertical or horizontal axis, which may result in a gastric outlet obstruction of variable degrees with potential ischemia, strangulation, perforation, and sepsis [5]. Early diagnosis of gastric volvulus is of paramount importance as it can lead to a substantial mortality risk if it was not recognized and managed appropriately [6].

As in the present case, gastric volvulus often develops after the fifth decade. Other risk factors associated with the development of gastric volvulus include diaphragmatic eventration, kyphoscoliosis, paraesophageal hernia, hiatal hernia, or phrenic nerve palsy [5]. In the current case, the patient was not known to have a prior history of gastroesophageal reflux disease. Gastric volvulus can be primary or secondary. In the primary form, the volvulus develops due to abnormalities in the gastric ligaments that may result from malignancy, adhesions, or kyphoscoliosis. However, gastric volvulus is more commonly secondary to other anatomic abnormalities like esophageal hernia [7].

The clinical manifestation of gastric volvulus may include acute or chronic presentations. It may present with abdominal pain or chest pain. Further, as in the present case, hematemesis may develop due to mucosal tears resulting from the severe vomiting episodes [5]. It is reported that up to $70 \%$ of patients with gastric volvulus present with the triad of pain, vomiting, and inability to pass the nasogastric tube, which is referred to as Borchardt's triad [6]. However, considering its rarity, gastric volvulus is not considered as the underlying etiology of such symptoms. The initial laboratory investigation could suggest evidence of volume depletion and electrolyte disturbances.

The definitive treatment of gastric volvulus is immediate or delayed surgical management. The surgery can be delayed in case the nasogastric decompression was not successful [8]. In the present case, the laparoscopic approach was performed as the patient was hemodynamically stable with no metabolic derangements.

\section{Conclusions}

Acute gastric volvulus is a very rare etiology of chest pain. Despite its rarity, physicians should keep a high index of suspicion of this condition after excluding the possible cardiac causes of chest pain. A computed tomography scan can make the diagnosis accurately. Laparoscopic treatment is a feasible and safe option for the management of acute gastric volvulus.

\section{Additional Information \\ Disclosures}

Human subjects: Consent was obtained or waived by all participants in this study. University Institutional Review Board issued approval N/A. The case report was waived by the institutional review board approval. Conflicts of interest: In compliance with the ICMJE uniform disclosure form, all authors declare the following: Payment/services info: All authors have declared that no financial support was received from any organization for the submitted work. Financial relationships: All authors have declared that they have no financial relationships at present or within the previous three years with any organizations that might have an interest in the submitted work. Other relationships: All authors have declared that there are no other relationships or activities that could appear to have influenced the submitted work.

\section{References}

1. Foy AJ, Filippone L: Chest pain evaluation in the emergency department . Med Clin North Am. 2015, 99:83547. 10.1016/j.mcna.2015.02.010

2. Lindsell CJ, Anantharaman V, Diercks D, et al.: The Internet Tracking Registry of Acute Coronary Syndromes (i*trACS): a multicenter registry of patients with suspicion of acute coronary syndromes reported using the standardized reporting guidelines for emergency department chest pain studies. Ann Emerg Med. 2006, 48:666-77. 10.1016/j.annemergmed.2006.08.005

3. Katelaris PH: Chest pain. Differentiating GIT from cardiac causes . Aust Fam Physician. 2001, 30:847-51.

4. Fass R, Dickman R: Non-cardiac chest pain: an update. Neurogastroenterol Motil. 2006, 18:408-17. 10.1111/j.1365-2982.2006.00787.x

5. Rashid F, Thangarajah T, Mulvey D, Larvin M, Iftikhar SY: A review article on gastric volvulus: a challenge to diagnosis and management. Int J Surg. 2010, 8:18-24. 10.1016/j.ijsu.2009.11.002

6. Chau B, Dufel S: Gastric volvulus. Emerg Med J. 2007, 24:446-7. 10.1136/emj.2006.041947

7. Shivanand G, Seema S, Srivastava DN, et al.: Gastric volvulus: acute and chronic presentation . Clin Imaging. 2003, 27:265-8. 10.1016/s0899-7071(02)00549-1

8. Coleman C, Musgrove K, Bardes J, et al.: Incarcerated paraesophageal hernia and gastric volvulus: Management options for the acute care surgeon, an Eastern Association for the Surgery of Trauma master 


\section{Cureus}

class video presentation. J Trauma Acute Care Surg. 2020, 88:e146-8. 10.1097/TA.0000000000002651 